\title{
EIGENVALUE COMPARISONS FOR BOUNDARY VALUE PROBLEMS OF THE DISCRETE BEAM EQUATION
}

\author{
JUN JI AND BO YANG
}

Received 29 September 2005; Revised 10 February 2006; Accepted 24 February 2006

We study the behavior of all eigenvalues for boundary value problems of fourth-order difference equations $\Delta^{4} y_{i}=\lambda a_{i+2} y_{i+2},-1 \leq i \leq n-2, y_{0}=\Delta^{2} y_{-1}=\Delta y_{n}=\Delta^{3} y_{n-1}=0$, as the sequence $\left\{a_{i}\right\}_{i=1}^{n}$ varies. A comparison theorem of all eigenvalues is established for two sequences $\left\{a_{i}\right\}_{i=1}^{n}$ and $\left\{b_{i}\right\}_{i=1}^{n}$ with $a_{j} \geq b_{j}, 1 \leq j \leq n$, and the existence of positive eigenvector corresponding to the smallest eigenvalue of the problem is also obtained in this paper.

Copyright (c) $2006 \mathrm{~J}$. Ji and B. Yang. This is an open access article distributed under the Creative Commons Attribution License, which permits unrestricted use, distribution, and reproduction in any medium, provided the original work is properly cited.

\section{Introduction}

Boundary value problems have important applications to physics, chemistry, and biology. For example, the boundary value problem

$$
\begin{aligned}
& u^{(4)}(t)=g(t) f(u(t)), \quad 0 \leq t \leq 1, \\
& u(0)=u^{\prime \prime}(0)=u^{\prime}(1)=u^{\prime \prime \prime}(1)=0
\end{aligned}
$$

arises in the study of elasticity and has definite physical meanings. Equation (1.1) is often referred to as the beam equation. It describes the deflection of a beam under a certain force. The boundary condition (1.2) means that the beam is simply supported at the end $t=0$ and fastened with a sliding clamp at $t=1$. In 2000, Graef and Yang [4] studied the problem (1.1)-(1.2), and obtained sufficient conditions for existence and nonexistence of positive solutions to the problem.

In this paper, we consider the eigenvalue problems for boundary value problems of fourth-order difference equations:

$$
\begin{gathered}
\Delta^{4} y_{i}=\lambda a_{i+2} y_{i+2}, \quad-1 \leq i \leq n-2, \\
y_{0}=\Delta^{2} y_{-1}=\Delta y_{n}=\Delta^{3} y_{n-1}=0,
\end{gathered}
$$

Hindawi Publishing Corporation Advances in Difference Equations Volume 2006, Article ID 81025, Pages 1-9 DOI 10.1155/ADE/2006/81025 
2 Eigenvalue comparisons for the beam equation

$$
\begin{gathered}
\Delta^{4} y_{i}=\mu b_{i+2} y_{i+2}, \quad-1 \leq i \leq n-2, \\
y_{0}=\Delta^{2} y_{-1}=\Delta y_{n}=\Delta^{3} y_{n-1}=0,
\end{gathered}
$$

where $\lambda$ and $\mu$ are parameters, and the forward difference operator $\Delta$ is defined as

$$
\Delta y_{i}=y_{i+1}-y_{i}
$$

We are going to show that comparison results can be established for all the eigenvalues of the systems (1.3) and (1.4) under certain conditions. Note that the problems (1.3) and (1.4) are discrete analogies to the following boundary value problems for fourth-order linear beam equations:

$$
\begin{array}{ll}
y^{(4)}(t)=\lambda a(t) y(t), & y(0)=y^{\prime \prime}(0)=y^{\prime}(1)=y^{\prime \prime \prime}(1)=0, \\
y^{(4)}(t)=\mu b(t) y(t), & y(0)=y^{\prime \prime}(0)=y^{\prime}(1)=y^{\prime \prime \prime}(1)=0 .
\end{array}
$$

Throughout the paper, we assume that

(H1) $n \geq 3$ is a fixed integer;

(H2) $a_{i} \geq 0$ and $b_{i} \geq 0$ for $1 \leq i \leq n$ with $\sum_{i=1}^{n} a_{i}>0$ and $\sum_{i=1}^{n} b_{i}>0$.

If $\lambda$ is a number (maybe complex) such that (1.3) has a nontrivial solution $\left\{y_{i}\right\}_{i=-1}^{n+2}$, then $\lambda$ is said to be an eigenvalue of the problem (1.3), and the corresponding nontrivial solution $\left\{y_{i}\right\}_{i=-1}^{n+2}$ is called an eigenvector of (1.3) corresponding to $\lambda$. Similarly, if $\mu$ is a number such that (1.4) has a nontrivial solution $\left\{y_{i}\right\}_{i=-1}^{n+2}$, then $\mu$ is said to be an eigenvalue of the problem (1.4), and the corresponding nontrivial solution $\left\{y_{i}\right\}_{i=-1}^{n+2}$ is called an eigenvector of (1.4) corresponding to $\mu$.

Travis [7] established some comparison results for the smallest eigenvalues of two eigenvalue problems for boundary value problems of $2 n$ th-order linear differential equations, by using the theory of $\boldsymbol{u}_{0}$-positive linear operator in a Banach space equipped with a cone of "nonnegative" elements. Since then, some progress has been made on comparisons of eigenvalues of boundary value problems of differential equations or difference equations. We refer the reader to the papers of Davis et al. [1], Gentry and Travis [2, 3], Hankerson and Peterson $[5,6]$. However, in all the papers mentioned above, the comparison results are for the smallest eigenvalues only.

The purpose of this paper is to establish the existence and comparison theorems for all the eigenvalues of the problems (1.3) and (1.4). We will also prove the existence of positive eigenvectors corresponding to the smallest eigenvalues of the problems.

\section{Eigenvalue comparisons}

In this section, we denote by $x^{*}$ the conjugate transpose of a vector $x$. A Hermitian matrix $A$ is said to be positive semidefinite if $x^{*} A x \geq 0$ for any $x$. It is said to be positive definite if $x^{*} A x>0$ for any nonzero $x$. In what follows we will write $X \geq Y$ if $X$ and $Y$ are Hermitian matrices of order $n$ and $X-Y$ is positive semidefinite. A matrix is said to be positive if every component of the matrix is positive. We also denote by $\operatorname{Nul}(X)$ the null space of a matrix $X$. 
The boundary conditions in (1.3) are the same as

$$
y_{0}=0, \quad y_{-1}=-y_{1}, \quad y_{n+1}=y_{n}, \quad y_{n+2}=y_{n-1}
$$

And the problem (1.3) is equivalent to the linear system

$$
(-D+\lambda A) y=0
$$

where $A=\operatorname{diag}\left(a_{1}, a_{2}, \ldots, a_{n-1}, a_{n}\right), y=\left(y_{1}, y_{2}, \ldots, y_{n-1}, y_{n}\right)^{T}$, and $D$ is a banded $n \times n$ matrix given by

$$
D=\left(\begin{array}{rrrrrrrrrr}
5 & -4 & 1 & 0 & 0 & \ldots & 0 & 0 & 0 & 0 \\
-4 & 6 & -4 & 1 & 0 & \ldots & 0 & 0 & 0 & 0 \\
1 & -4 & 6 & -4 & 1 & \ldots & 0 & 0 & 0 & 0 \\
0 & 1 & -4 & 6 & -4 & \ldots & 0 & 0 & 0 & 0 \\
\ldots & \ldots & \ldots & \ldots & \ldots & \ldots & \ldots & \ldots & \ldots & \ldots \\
0 & 0 & 0 & 0 & \ldots & 1 & -4 & 6 & -4 & 1 \\
0 & 0 & 0 & 0 & \ldots & 0 & 1 & -4 & 6 & -3 \\
0 & 0 & 0 & 0 & \ldots & 0 & 0 & 1 & -3 & 2
\end{array}\right) .
$$

Obviously, there is a one-to-one correspondence between the solution

$$
\left(y_{1}, y_{2}, \ldots, y_{n-1}, y_{n}\right)^{T}
$$

to the problem (2.2) and the solution $\left(y_{-1}, y_{0}, y_{1}, \ldots, y_{n}, y_{n+1}, y_{n+2}\right)^{T}$ to the problem (1.3) under the relationship (2.1). We will not distinguish one from the other, denote by $y$ either one of these two vectors, and say that problems (1.3) and (2.2) are equivalent. Similarly, the problem (1.4) is equivalent to

$$
(-D+\mu B) y=0
$$

where $B=\operatorname{diag}\left(b_{1}, b_{2}, \ldots, b_{n-1}, b_{n}\right)$ and $D, y$ are defined as above.

Let $e_{i}$ be the $i$ th column of the identity matrix $I$ of order $n$. Define the elementary matrix $P_{i}=I+e_{i-1} e_{i}^{T}$. It is easily seen that $A P_{i}=A+\left(A e_{i-1}\right) e_{i}^{T}$ is the matrix obtained by adding the $(i-1)$ th column of $A$ to the $i$ th column of $A$ and that $P_{i} A=A+e_{i-1}\left(e_{i}^{T} A\right)$ is the matrix obtained by adding the $i$ th row of $A$ to the $(i-1)$ th row of $A$. Similarly, $A P_{i}^{T}$ and $P_{i}^{T} A$ are matrices obtained by adding the $i$ th column of $A$ to the $(i-1)$ th column of $A$ and by adding the $(i-1)$ th row of $A$ to the $i$ th row of $A$, respectively.

Lemma 2.1. $D$ is positive definite and $D^{-1}$ is a positive matrix.

Proof. Multiplying on $D$ by $P_{n}$ and $P_{n}^{T}$, we have $P_{n} D P_{n}^{T}$. Further multiplying $P_{n-1}$ and $P_{n-1}^{T}$, we have $P_{n-1} P_{n} D P_{n}^{T} P_{n-1}^{T}$, continuing this way, $P_{2} P_{3} \cdots P_{n-1} P_{n} D P_{n}^{T} P_{n-1}^{T} \cdots P_{3}^{T} P_{2}^{T}$ will be a tridiagonal matrix which is further reduced to the identity matrix by multiplying $P_{2}^{T}$ and $P_{2}, P_{3}^{T}$ and $P_{3}, \ldots, P_{n}^{T}$ and $P_{n}$, that is,

$$
P_{n}^{T} P_{n-1}^{T} \cdots P_{3}^{T} P_{2}^{T} P_{2} P_{3} \cdots P_{n-1} P_{n} D P_{n}^{T} P_{n-1}^{T} \cdots P_{3}^{T} P_{2}^{T} P_{2} P_{3} \cdots P_{n-1} P_{n}=I
$$


4 Eigenvalue comparisons for the beam equation

Thus, we have

$$
D=W^{T} W
$$

where

$$
W=P_{n}^{-1} P_{n-1}^{-1} \cdots P_{3}^{-1} P_{2}^{-1} P_{2}^{-T} P_{3}^{-T} \cdots P_{n-1}^{-T} P_{n}^{-T}
$$

Obviously, $D$ is positive definite since $W$ is nonsingular. We also have

$$
D^{-1}=P_{n}^{T} P_{n-1}^{T} \cdots P_{3}^{T} P_{2}^{T} P_{2} P_{3} \cdots P_{n-1} P_{n} P_{n}^{T} P_{n-1}^{T} \cdots P_{3}^{T} P_{2}^{T} P_{2} P_{3} \cdots P_{n-1} P_{n},
$$

which is positive due to the fact that each $P_{i}(2 \leq i \leq n)$ is positive. The proof is complete.

Lemma 2.2. If $\lambda$ is an eigenvalue of the problem (1.3) and $y=\left(y_{1}, y_{2}, \ldots, y_{n-1}, y_{n}\right)^{T}$ is a corresponding eigenvector, then

(a) $y^{*}$ Ay $>0$,

(b) $\lambda$ is real and positive,

(c) if $\rho$ is an eigenvalue of the problem (1.3) which is different from $\lambda$ and $x=\left(x_{1}, x_{2}, \ldots\right.$, $\left.x_{n-1}, x_{n}\right)^{T}$ is a corresponding eigenvector, then $x^{T} A y=0$.

Proof. (a) The assumption (H2) indicates that $y^{*} A y \geq 0$. Assume the contrary, that $y^{*} A y=0$. Obviously, we have $\sqrt{A} y=0$ where $\sqrt{A}=\operatorname{diag}\left(\sqrt{a_{1}}, \sqrt{a_{2}}, \ldots, \sqrt{a_{n}}\right)$. Then $D y=$ $\lambda A y=\lambda \sqrt{A} \sqrt{A} y=0$ which, as well as Lemma 2.1, implies that $y=0$, a contradiction.

(b) We can write

$$
\lambda y^{*} A y=y^{*}(\lambda A y)=y^{*} D y=y^{*} D^{*} y=(D y)^{*} y=(\lambda A y)^{*} y=\bar{\lambda} y^{*} A^{*} y=\bar{\lambda} y^{*} A y,
$$

which, together with (a), implies that $\lambda=\bar{\lambda}$, that is, $\lambda$ is real. Finally, the relations above indicate that $\lambda=y^{*} D y /\left(y^{*} A y\right)>0$ thanks to Lemmas 2.1 and 2.2(a).

The part (c) follows from

$$
(\lambda-\rho) x^{T} A y=\lambda x^{T} A y-\rho x^{T} A y=x^{T}(\lambda A y)-(\rho A x)^{T} y=x^{T} D y-(D x)^{T} y=0 .
$$

The proof is complete.

Lemma 2.3. The eigenvalues of the problem (1.3) are related to those of the matrix $D^{-1 / 2} A D^{-1 / 2}$ as follows.

(a) If $\lambda$ is an eigenvalue of the problem (1.3), then $1 / \lambda$ is an eigenvalue of $D^{-1 / 2} A D^{-1 / 2}$.

(b) If $\alpha$ is a positive eigenvalue of $D^{-1 / 2} A D^{-1 / 2}$, then $1 / \alpha$ is an eigenvalue of the problem (1.3). 
Proof. (a) Let $\lambda$ be an eigenvalue of the problem (1.3) and $y=\left(y_{1}, y_{2}, \ldots, y_{n-1}, y_{n}\right)^{T}$ be a corresponding eigenvector. Then, in view of Lemma 2.2, $\lambda>0$ and $\lambda A y=D y$. Therefore

$$
\begin{gathered}
\lambda A y=D^{1 / 2} D^{1 / 2} y, \\
D^{-1 / 2} A D^{-1 / 2}\left(D^{1 / 2} y\right)=\frac{1}{\lambda}\left(D^{1 / 2} y\right) .
\end{gathered}
$$

The result in (b) can be proved similarly. The proof is complete.

Next, we state the well-known Perron-Frobenius theorem. For a proof, please refer to [8, page 30$]$.

Lemma 2.4 (Perron-Frobenius). Let $A$ be a real square matrix. If $A$ is also a nonnegative irreducible matrix, then the spectral radius $\rho(A)$ of the matrix $A$ is a simple eigenvalue of $A$ associated to a positive eigenvector. Moreover, $\rho(A)>0$.

THEOREM 2.5. If $\lambda_{1}>0$ is the smallest eigenvalue of the problem (1.3), then there exists a positive eigenvector $y$ corresponding to $\lambda_{1}$.

Proof. We note that

$$
D^{-1} A y=\frac{1}{\lambda_{1}} y
$$

Thus $1 / \lambda_{1}$ is the maximum eigenvalue of $D^{-1} A$ and the $y$ is an eigenvector corresponding to $1 / \lambda_{1}$.

In the case when $a_{i}>0$ for all $1 \leq i \leq n$, we obtain that the matrix $D^{-1} A$ is positive in view of Lemma 2.1 and thus is irreducible. Therefore, the result follows immediately from the Perron-Frobenius theorem.

In the case when some of the $a_{i}$ 's are zero, without loss of generality we assume that $a_{1}=a_{2}=\cdots=a_{p}=0$ and $a_{i}>0$ for $p<i \leq n$, we can write $D^{-1} A$ as follows:

$$
D^{-1} A=\left(\begin{array}{ll}
O & V \\
O & Z
\end{array}\right),
$$

where $V$ is a $p \times(n-p)$ matrix and $Z$ is a $(n-p) \times(n-p)$ matrix. Both $V$ and $Z$ are positive matrices. Also, $1 / \lambda_{1}$ is the maximum eigenvalue of $Z$. Applying the Perron-Frobenius theorem to the positive matrix $Z$, there exists a positive vector $y_{z}>0$ such that

$$
Z y_{z}=\frac{1}{\lambda_{1}} y_{z}
$$

Define $y_{v}=\lambda_{1} V y_{z}$ and $y=\left(y_{v}^{T}, y_{z}^{T}\right)^{T}$. Obviously, we have

$$
y>0, \quad D^{-1} A y=\frac{1}{\lambda_{1}} y .
$$

This completes the proof.

Lemma 2.6. Suppose that $z=\left(z_{-1}, z_{0}, z_{1}, \ldots, z_{n+2}\right)^{T}$ is a nonzero solution to (1.3). Then $z_{1} \neq 0$. 
6 Eigenvalue comparisons for the beam equation

Proof. Assume the contrary, that is, $z_{1}=0$. Then, it is easily seen from the initial conditions in (1.3) that

$$
\Delta z_{0}=z_{1}-z_{0}=0, \quad \Delta z_{-1}=\Delta z_{0}-\Delta^{2} z_{-1}=0, \quad z_{-1}=z_{0}-\Delta z_{-1}=0 .
$$

We claim that $z_{2}=0$. Assume the contrary, that is, $z_{2} \neq 0$. For simplicity, we rescale the vector $z$ so that $z_{2}=1$. Therefore, it is seen from (1.3) and (2.17) that

$$
\Delta z_{1}=z_{2}-z_{1}=1, \quad \Delta^{2} z_{0}=\Delta z_{1}-\Delta z_{0}=1, \quad \Delta^{3} z_{-1}=\Delta^{2} z_{0}-\Delta^{2} z_{-1}=1,
$$

which further implies that

$$
\begin{gathered}
\Delta^{4} z_{-1}=\lambda a_{1} z_{1} \geq 0, \\
\Delta^{3} z_{0}=\Delta^{3} z_{-1}+\Delta^{4} z_{-1} \geq 1+0 \geq 1, \\
\Delta^{2} z_{1}=\Delta^{2} z_{0}+\Delta^{3} z_{0} \geq 1+1 \geq 1, \\
\Delta z_{2}=\Delta z_{1}+\Delta^{2} z_{1} \geq 1+1 \geq 1, \\
z_{3}=z_{2}+\Delta z_{2} \geq 1+1 \geq 1 .
\end{gathered}
$$

Similarly, we have

$$
\Delta^{4} z_{0}=\lambda a_{2} z_{2} \geq 0, \quad \Delta^{3} z_{1} \geq 1, \quad \Delta^{2} z_{2} \geq 1, \quad \Delta z_{3} \geq 1, \quad z_{4} \geq 1 .
$$

Continuing this procedure, we will finally get

$$
\Delta^{4} z_{n-2}=\lambda a_{n} z_{n} \geq 0, \quad \Delta^{3} z_{n-1} \geq 1, \quad \Delta^{2} z_{n} \geq 1, \quad \Delta z_{n+1} \geq 1, \quad z_{n+2} \geq 1,
$$

which contradicts the boundary condition $\Delta^{3} z_{n-1}=0$. Therefore, we have $z_{-1}=z_{0}=z_{1}=$ $z_{2}=0$. The difference equation (1.3) can be written as

$$
y_{i+4}=4 y_{i+3}+\left(\lambda a_{i+2}-6\right) y_{i+2}+4 y_{i+1}-y_{i}, \quad-1 \leq i \leq n-2,
$$

from which $z_{i}=0$ for all $i$ can be deduced recursively from the initial conditions $z_{-1}=$ $z_{0}=z_{1}=z_{2}=0$. This contradicts the assumption that $z \neq 0$. Therefore we have $z_{1} \neq 0$.

Lemma 2.7. Suppose that both

$$
x=\left(x_{-1}, x_{0}, x_{1}, \ldots, x_{n+2}\right)^{T}, \quad y=\left(y_{-1}, y_{0}, y_{1}, \ldots, y_{n+2}\right)^{T}
$$

are nonzero solutions to (1.3) for a fixed $\lambda$. Then $x$ and $y$ are linearly dependent.

Proof. It is easily seen from Lemma 2.6 that $x_{1} \neq 0$ and $y_{1} \neq 0$. Define $z=y_{1} x-x_{1} y$. Obviously, $z$ is a solution to (1.3) with $z_{1}=0$. Therefore, in view of Lemma 2.6, $z$ is a trivial solution, that is, $z=0$, leading to the desired result. 
Lemma 2.8. Let $N \geq 1$ be the number of positive elements in the set $\left\{a_{1}, a_{2}, \ldots, a_{n}\right\}$. Then there are $N$ distinct eigenvalues $\lambda_{i}(i=1,2, \ldots, N)$ of the problem (1.3) and $\alpha_{i}=1 / \lambda_{i}(i=$ $1,2, \ldots, N)$ are the only positive eigenvalues of $D^{-1 / 2} A D^{-1 / 2}$.

Proof. The assumption (H2) implies $N \geq 1$. Suppose that $\alpha_{1} \geq \alpha_{2} \geq \cdots \geq \alpha_{n} \geq 0$ are all eigenvalues of $D^{-1 / 2} A D^{-1 / 2}$. The fact that $D^{-1 / 2} A D^{-1 / 2}$ is real and symmetric indicates that there exists an orthogonal matrix $Q$ such that

$$
Q^{T} D^{-1 / 2} A D^{-1 / 2} Q=\operatorname{diag}\left(\alpha_{1}, \alpha_{2}, \ldots, \alpha_{n}\right)
$$

The nonsingularity of $D^{-1 / 2} Q$ and (2.24) imply that

$$
\operatorname{rank}(A)=\operatorname{rank}\left(Q^{T} D^{-1 / 2} A D^{-1 / 2} Q\right)=\operatorname{rank}\left(\operatorname{diag}\left(\alpha_{1}, \alpha_{2}, \ldots, \alpha_{n}\right)\right)
$$

indicating that the number of positive $\alpha_{i}$ is the same as that of positive $a_{i}$ in $A$ which is equal to $N$.

We claim that all of positive $\alpha_{i}, i=1,2, \ldots, N$, are distinct. Suppose the contrary that $\alpha_{i_{0}}=\alpha_{i_{0}+1}>0$ for some $i_{0}$ where $1 \leq i_{0} \leq N-1$. Observe that $Q^{T} D^{-1 / 2} A D^{-1 / 2} Q e_{i}=\alpha_{i} e_{i}$ (see (2.24)) which further implies that

$$
D\left(D^{-1 / 2} Q e_{i}\right)=\frac{1}{\alpha_{i}} A\left(D^{-1 / 2} Q e_{i}\right), \quad i=i_{0}, i_{0}+1
$$

Thus, we have two independent nonzero solutions to (2.2) with $\lambda=1 / \alpha_{i_{0}}$ from which two independent nonzero solutions to (1.3) with $\lambda=1 / \alpha_{i_{0}}$ can be constructed, contradicting Lemma 2.7. Thus, it is seen from Lemma 2.3 that $\left\{\lambda_{i}=1 / \alpha_{i}: i=1,2, \ldots, N\right\}$ gives the complete set of eigenvalues of the problem (1.3). The proof is complete.

Theorem 2.9. Assume the hypotheses of (H1)-(H2) hold. Let $j$ be the number of positive elements in the set $\left\{a_{1}, a_{2}, \ldots, a_{n}\right\}$ and let $k$ be the number of positive elements in the set $\left\{b_{1}, b_{2}, \ldots, b_{n}\right\}$. Let $\left\{\lambda_{1}<\lambda_{2}<\cdots<\lambda_{j}\right\}$ be the set of all eigenvalues of the problem (1.3) and let $\left\{\mu_{1}<\mu_{2}<\cdots<\mu_{k}\right\}$ be the set of all eigenvalues of the problem (1.4). If $a_{i} \geq b_{i}$ for $1 \leq i \leq n$, then $\lambda_{i} \leq \mu_{i}$ for $1 \leq i \leq k$.

Proof. In view of Lemma 2.8, we have that

$$
\begin{array}{ll}
\alpha_{1}=\frac{1}{\lambda_{1}}>\alpha_{2}=\frac{1}{\lambda_{2}}>\cdots>\alpha_{j}=\frac{1}{\lambda_{j}}>0, & \alpha_{j+1}=\cdots=\alpha_{n}=0, \\
\beta_{1}=\frac{1}{\mu_{1}}>\beta_{2}=\frac{1}{\mu_{2}}>\cdots>\beta_{k}=\frac{1}{\mu_{k}}>0, & \beta_{k+1}=\cdots=\beta_{n}=0
\end{array}
$$

are the eigenvalues of $D^{-1 / 2} A D^{-1 / 2}$ and $D^{-1 / 2} B D^{-1 / 2}$, respectively. If $a_{i} \geq b_{i}$ for $1 \leq i \leq n$, then $A \geq B$ implying that

$$
D^{-1 / 2} A D^{-1 / 2} \geq D^{-1 / 2} B D^{-1 / 2}
$$


8 Eigenvalue comparisons for the beam equation

By Weyl's inequality and (2.28), we have

$$
\alpha_{i} \geq \beta_{i} \geq 0, \quad 1 \leq i \leq n .
$$

The desired result follows directly from (2.27) and (2.29). The proof is complete.

Remark 2.10. Equation (1.1) is usually studied together with a set of boundary conditions, which might be (1.2) or one of the following:

$$
\begin{aligned}
& u(0)=u^{\prime \prime}(0)=u^{\prime}(1)=u(1)=0, \\
& u(0)=u^{\prime \prime}(0)=u^{\prime \prime}(1)=u(1)=0, \\
& u(0)=u^{\prime}(0)=u^{\prime}(1)=u^{\prime \prime \prime}(1)=0, \\
& u(0)=u^{\prime}(0)=u^{\prime \prime}(1)=u^{\prime \prime \prime}(1)=0, \\
& u(0)=u^{\prime}(0)=u^{\prime}(1)=u(1)=0 .
\end{aligned}
$$

Each of the above boundary conditions has specific physical meaning. For example, (2.30) means that the beam is simply supported at the end $t=0$, and embedded at the end $t=1$.

In fact, the results obtained in this paper can be generalized to eigenvalue problems of boundary value problems of linear beam equations which include the discrete form of any of the above boundary conditions, (2.30) through (2.34). For example, comparison results can be established for the eigenvalue problems for boundary value problems of fourth-order difference equations

$$
\begin{gathered}
\Delta^{4} y_{i}=\lambda a_{i+2} y_{i+2}, \quad-1 \leq i \leq n-2, \\
y_{-1}=\Delta y_{-1}=\Delta y_{n+1}=y_{n+2}=0, \\
\Delta^{4} y_{i}=\mu b_{i+2} y_{i+2}, \quad-1 \leq i \leq n-2, \\
y_{-1}=\Delta y_{-1}=\Delta y_{n+1}=y_{n+2}=0,
\end{gathered}
$$

where $y_{-1}=\Delta y_{-1}=\Delta y_{n+1}=y_{n+2}=0$ is the discrete form of (2.34). We leave the details of such generalizations to the reader.

\section{References}

[1] J. M. Davis, P. W. Eloe, and J. Henderson, Comparison of eigenvalues for discrete Lidstone boundary value problems, Dynamic Systems and Applications 8 (1999), no. 3-4, 381-388.

[2] R. D. Gentry and C. C. Travis, Comparison of eigenvalues associated with linear differential equations of arbitrary order, Transactions of the American Mathematical Society 223 (1976), 167179.

[3] ___ Existence and comparison of eigenvalues of $n$th order linear differential equations, Bulletin of the American Mathematical Society 82 (1976), no. 2, 350-352.

[4] J. R. Graef and B. Yang, Existence and nonexistence of positive solutions of fourth order nonlinear boundary value problems, Applicable Analysis 74 (2000), no. 1-2, 201-214.

[5] D. Hankerson and A. Peterson, Comparison theorems for eigenvalue problems for nth order differential equations, Proceedings of the American Mathematical Society 104 (1988), no. 4, 12041211. 
[6] Comparison of eigenvalues for focal point problems for nth order difference equations, Differential and Integral Equations 3 (1990), no. 2, 363-380.

[7] C. C. Travis, Comparison of eigenvalues for linear differential equations of order $2 n$, Transactions of the American Mathematical Society 177 (1973), 363-374.

[8] R. S. Varga, Matrix Iterative Analysis, Prentice-Hall, New Jersey, 1962.

Jun Ji: Department of Mathematics, Kennesaw State University, Kennesaw, GA 30144, USA

E-mail address: jji@kennesaw.edu

Bo Yang: Department of Mathematics, Kennesaw State University, Kennesaw, GA 30144, USA

E-mail address: byang@kennesaw.edu 